\title{
ASPECTOS EPIDEMIOLÓGICOS E HEMATOLÓGICOS DE CÃES INFECTADOS COM EHRLICHIA SP E ANAPLASMA SP EM JATAÍ-GO, BRASIL.
}

\author{
Ariel Eurides Stella ${ }^{1}$ \\ Vera Lúcia Dias da Silva ${ }^{1}$ \\ Cecília Nunes Moreira ${ }^{1}$ \\ Sidney Aniceto Rezende Júnior ${ }^{2}$ \\ Dalila Almeida Lima ${ }^{3}$ \\ Micael Siegert Schimmunech ${ }^{4}$
}

\begin{abstract}
RESUMO
No Brasil, as infecções por hemoparasitas representam uma das principais causas de busca por assistência veterinária em cães. Infecções por bactérias da ordem Rickettsiales que incluem Ehrlichia sp. e Anaplasma sp. tem uma grande participação nessas buscas. Foi objetivo deste estudo elucidar aspectos epidemiológicos regionais e hematológicos de infecções por Anaplasma sp. e Ehrlichia sp, muitas vezes assintomáticas, em cães no município de Jataí-GO. Os dados deste estudo foram baseados em amostras de sangue de cães atendidos no Hospital Veterinário da Universidade Federal de Jataí, em Jataí, Goiás, entre os anos de 2005 e 2017. A observação microscópica da presença de mórulas de Ehrlichia sp. e Anaplasma sp. foi utilizada como critério de positividade. 5.015 animais foram atendidos nesse período e $691(13,78 \%)$ deles foram positivos para a presença destes hemoparasitas. Entre esses animais, 9,23\% (463/5015) possuíam mórulas de Ehrlichia sp., e 4,37\% (219/5015) apresentavam mórulas típicas de Anaplasma sp., enquanto 0,18\% (9/5015) possuíam mórula de ambas as espécies. As fêmeas representaram 57,89\% (400/691) dos animais positivos e os machos 42,11\% (291/691). 35,02\% (242/691) dos animais tinham menos de 1 ano de vida, 54,13\% (374/691) tinham entre 1 e 8 anos, e 10,85\% (75/691) tinham mais de 8 anos. As fêmeas, com menos de um ano de idade e coinfectadas, apresentaram as alterações mais significativas nos aspectos hematológicos, hematócrito (29\%), contagem de hemácias (3 milhões $/ \mathrm{mm}^{3}$ ), contagem de plaquetas $\left(40.000 / \mathrm{mm}^{3}\right)$ e contagem total de leucócitos $\left(16.000 \mathrm{mil} / \mathrm{mm}^{3}\right)$, evidenciando anemia e trombocitopenia. Animais infectados com Anaplasma sp. e Ehrlichia sp., ao mesmo tempo, apresentaram trombocitopenia mais intensa, evidenciada em machos entre 1 e 8 anos $\left(30.000 / \mathrm{mm}^{3}\right)$. As infecções por Anaplasma sp. causaram uma diminuição severa na média da contagem de glóbulos vermelhos, evidenciada em machos (3 milhões $\left./ \mathrm{mm}^{3}\right)$ e fêmeas com menos de 1 ano $\left(3,79\right.$ milhões $\left./ \mathrm{mm}^{3}\right)$.
\end{abstract}

Palavras-chave: erliquiose, mórula, carrapato.

\section{EPIDEMIOLOGICAL AND HEMATOLOGICAL ASPECTS OF DOGS WITH EHRLICHIA SP AND ANAPLASMA SP IN JATAÍ-GO.}

\begin{abstract}
In Brazil, infections by hemoparasites represent one of the main causes of seeking veterinary assistance in dogs. Infections with bacteria of the order Rickettsiales that include Ehrlichia sp.

\footnotetext{
${ }^{1}$ Professor Associado Universidade Federal de Jataí - UFJ - Correspondência: ariel.vet@gmail.com

${ }^{2}$ Técnico Laboratório de Análises Clínicas Veterinárias. Universidade Federal de Jataí - UFJ

${ }^{3}$ Mestranda no Programa de Pós-Graduação em Biociência Animal. Universidade Federal de Jataí - UFJ

${ }^{4}$ Discente do curso de Medicina Veterinária. Universidade Federal de Jataí - UFJ
}

Stella AE, Silva VLD, Moreira CN, Rezende Junior AS, Lima DA, Schimmunech MS. Aspectos epidemiológicos e hematológicos de cães infectados com Ehrlichia sp e Anaplasma SP em Jataí-GO, Brasil. Vet. e Zootec. 2021; v28: 001-011.
\end{abstract}


and Anaplasma sp. has a great participation in these assistances. The objective of this study was to elucidate regional epidemiologic and hematologic aspects of infections by Anaplasma sp. and Ehrlichia sp., often asymptomatic, in dogs in the municipality of Jataí-GO. The data in this study were based on blood samples from dogs treated at the Veterinary Hospital of the Federal University of Jataí, in Jataí, Goiás, between the years 2005 and 2017. Microscopic observation of the presence of Ehrlichia sp. and Anaplasma sp. was used as a positivity criterion. 5,015 animals were treated during this period and $691(13.78 \%)$ of them were positive for the presence of these hemoparasites. Among these animals, 9.23\% (463/5015) had morulae from Ehrlichia sp., and 4.37\% (219/5015) had morulae typical of Anaplasma sp., while $0.18 \%$ (9/5015) had morulae of both species. Females represented $57.89 \%$ (400/691) of positive animals and males $42.11 \%$ (291/691). 35.02\% (242/691) of the animals were less than one year old, 54.13\% (374/691) were between 1 and 8 years old, and 10.85\% (75/691) were over 8 years old. Females, less than one year old and co-infected, showed the most significant changes in hematological, hematocrit (29\%), red blood cell count (3 million / $\left.\mathrm{mm}^{3}\right)$, platelet count $\left(40,000 / \mathrm{mm}^{3}\right)$ and total blood count leukocytes (16,000 thousand / $\mathrm{mm}^{3}$ ), showing anemia and thrombocytopenia. Animals infected with Anaplasma sp. and Ehrlichia sp., at the same time, presented more intense thrombocytopenia, evidenced in males between 1 and 8 years old $\left(30,000 / \mathrm{mm}^{3}\right)$. Infections by Anaplasma sp. caused a severe decrease in the mean red blood cell count, evidenced in males $\left(3\right.$ million $\left./ \mathrm{mm}^{3}\right)$ and females under 1 year $\left(3.79\right.$ million $\left./ \mathrm{mm}^{3}\right)$.

Keywords: ehrlichiosis, morulae, tick.

\section{ASPECTOS EPIDEMIOLÓGICOS Y HEMATOLÓGICOS DE PERROS CON EHRLICHIA SP Y ANAPLASMA SP EN JATAÍ-GO.}

\section{RESUMEN}

En Brasil, las infecciones por hemoparásitos representan una de las principales causas de búsqueda de asistencia veterinaria en perros. Infecciones por bacterias del orden Rickettsiales que incluyen Ehrlichia sp. y Anaplasma sp. tienen una gran participación en estas búsquedas. El objetivo de este estudio fue dilucidar los aspectos epidemiológicos regionales y hematológicos de las infecciones por Anaplasma sp. y Ehrlichia sp., muchas veces asintomáticas, en perros del municipio de Jataí-GO. Los datos de este estudio se basaron en muestras de sangre de perros tratados en el Hospital Veterinario de la Universidad Federal de Jataí, en Jataí, Goiás, entre los años 2005 y 2017. Observación microscópica de la presencia de Ehrlichia sp. y Anaplasma sp. se utilizó como criterio de positividad. Durante este período se trataron 5.015 animales y $691(13,78 \%)$ de ellos resultaron positivos para la presencia de estos hemoparásitos. Entre estos animales, 9,23\% (463/5015) tenía mórulas de Ehrlichia sp., y 4,37\% (219/5015) tenían mórulas típicas de Anaplasma sp., mientras que 0,18\% (9/5015) tenían mórulas de ambas especies. Las hembras representaron 57,89\% (400/691) de los animales positivos y los machos 42,11\% (291/691). 35,02\% (242/691) de los animales tenían menos de 1 año, 54,13\% (374/691) tenían entre 1 y 8 años y 10,85\% (75/691) tenían más de 8 años. Las hembras, menores de un año y coinfectadas, mostraron los cambios más significativos en hematología, hematocrito (29\%), recuento de glóbulos rojos (3 millones / $\left.\mathrm{mm}^{3}\right)$, recuento de plaquetas $\left(40.000 / \mathrm{mm}^{3}\right)$ y recuento sanguíneo total leucocitos (16.000 mil Stella AE, Silva VLD, Moreira CN, Rezende Junior AS, Lima DA, Schimmunech MS. Aspectos epidemiológicos e hematológicos de cães infectados com Ehrlichia sp e Anaplasma SP em Jataí-GO, Brasil. Vet. e Zootec. 2021; v28: 001-011. 
$/ \mathrm{mm}^{3}$ ), mostrando anemia y trombocitopenia. Los animales infectados por Anaplasma sp. y Ehrlichia sp., al mismo tiempo, presentaron trombocitopenia más intensa, evidenciada en machos entre 1 y 8 años $\left(30.000 / \mathrm{mm}^{3}\right)$. Infecciones por Anaplasma sp. causó una disminución severa en el recuento promedio de glóbulos rojos, evidenciada en machos (3 millones $/ \mathrm{mm}^{3}$ ) y hembras menores de 1 año $\left(3.79\right.$ millones $\left./ \mathrm{mm}^{3}\right)$.

Palabras clave: ehrlichiosis, mórula, garrapata.

\section{INTRODUÇÃO}

Os gêneros Anaplasma sp. e Ehrlichia sp. pertencem à família Anaplasmataceae, que são bactérias Gram-negativas intracelulares, e se replicam dentro de vacúolos ligados a membrana das células (1). Infectam principalmente monócitos, macrófagos e linfócitos, formando agregados bacterianos intracitoplasmáticos, chamados mórulas (2). As espécies destes grupos são os agentes patogênicos, transmitidos por carrapatos Rhipicephalus sanguineus $(3,4)$, mais comuns em cães no mundo inteiro $(5,6,7)$. A distribuição e a transmissão geográfica estão principalmente relacionadas a este vetor (8). São os agentes causadores de condições patológicas chamadas de erliquiose monocítica canina (EMC) e trombocitopenia cíclica canina (TCC) (9). Nos últimos anos, as doenças transmitidas por carrapatos como erliquiose e anaplasmose tem se disseminado por todo o mundo, ameaçando a saúde de animais de companhia e humanos (4). Pois, devido a convivência próxima entre os humanos e seus cães, existem aspectos importantes relacionados a saúde pública. Além disso, Bowman et al. (10) citam que os cães podem servir como sentinelas, para identificar a presença de agentes transmitidos por carrapatos, que são importantes tanto para os animais como para humanos. Emiroglu et al. (11) ainda relatam o caso de uma infecção humana causada por E. canis.

O curso clínico da infecção pode ser dividido em fases aguda, subclínica e crônica (12). E ainda de acordo com Geromichalou e Faixová (8) não há predileção por idade ou sexo, podendo todas as raças serem infectadas. Já as alterações causadas pela $E$. canis incluem febre, letargia, claudicação, secreção oculonasal, trombocitopenia, anemia não regenerativa, leucopenia, hiperglobulinemia e proteinúria durante vários estágios de infecção. Sendo a trombocitopenia uma característica importante (13). O diagnóstico da infecção por estes hemoparasitas pode ser feito por vários métodos, como a demonstração intracitoplasmática das mórulas, cultura, sorologia e PCR, entretanto Nakaghi et al. (14) citam que os resultados destas técnicas devem sempre ser complementares ao exame clínico e hematológico, e que a sorologia tem um importante papel nas fases subclínica e crônica da doença.

A frequência regional dessas infecções é bastante variável, entretanto estas bactérias e seus vetores estão presentes no mundo todo. Monitorar a presença desses agentes, e as características clínicas dos animais infectados, é fundamental por permitir que médicos veterinários tenham uma melhor visão do quadro da doença em suas regiões, fomentando protocolos de tratamento e prevenção mais ajustados.

\section{MATERIAL E MÉTODOS}

Este estudo baseou-se na avaliação retrospectiva da casuística de atendimentos de cães do Hospital Veterinário da Universidade Federal de Jataí, em Jataí, GO, entre os anos de 2005 e 2017. Fez-se o levantamento de todos os casos clínicos de animais atendidos neste período $(\mathrm{n}=5015)$, discriminando-os em machos e fêmeas. Foram examinadas as fichas clínicas e Stella AE, Silva VLD, Moreira CN, Rezende Junior AS, Lima DA, Schimmunech MS. Aspectos epidemiológicos e hematológicos de cães infectados com Ehrlichia sp e Anaplasma SP em Jataí-GO, Brasil. Vet. e Zootec. 2021; v28: 001-011. 
laboratoriais dos cães que apresentavam mórulas de Ehrlichia sp. e Anaplasma sp. em leucócitos de esfregaços sanguíneos, confeccionados com sangue obtido por punção de vasos marginais do pavilhão auricular e corados pelo May-Grünwald-Giemsa. Dos 691 casos positivos, realizou-se uma análise descritiva dos seguintes dados: idade, sexo, data do atendimento, e quadro hematológico (eritrograma e leucograma). Foi calculada também a mediana dos valores hematológicos (hematócrito, hemácias, plaquetas e leucograma) de acordo com a faixa etária e sexo do paciente. Os autores confirmam que as políticas éticas da revista foram observadas. Nenhuma aprovação ética foi necessária, pois nenhuma experimentação animal foi conduzida neste estudo. Os autores também declaram não haver conflitos de interesse.

\section{RESULTADOS}

Entre o período de janeiro de 2005 até dezembro de 2017, 5.015 fichas de exames de análises clínicas veterinárias foram analisadas. Destas $691(13,78 \%)$ foram positivas para a presença destes hemoparasitas. Entre esses animais, 9,23\% (463/5015) possuíam mórulas de Ehrlichia sp., e 4,37\% (219/5015) apresentavam mórulas típicas de Anaplasma sp., enquanto $0,18 \%$ (9/5015) possuíam mórula de ambas as espécies (Tabela 1). As fêmeas representaram $57,89 \%$ (400/691) dos animais positivos e os machos 42,11\% (291/691) (Tabela 2). 35,02\% (242/691) dos animais tinham menos de 1 ano de vida, 54,13\% (374/691) tinham entre 1 e 8 anos, e 10,85\% (75/691) tinham mais de 8 anos (Tabela 3). Os valores hematológicos gerais estão presentes na Tabela 4. As fêmeas, com menos de um ano, infectadas com Ehrlichia sp. e Anaplasma sp. apresentaram as alterações mais significativas nos aspectos hematológicos, hematócrito (29\%), contagem de hemácias (3 milhões $/ \mathrm{mm}^{3}$ ), contagem de plaquetas $\left(40.000 / \mathrm{mm}^{3}\right)$ e contagem total de leucócitos $\left(16.000 \mathrm{mil} / \mathrm{mm}^{3}\right)$, evidenciando anemia e trombocitopenia (Tabela 5). Animais infectados com Anaplasma sp. e Ehrlichia sp. ao mesmo tempo, apresentaram trombocitopenia mais intensa, evidenciada em machos entre 1 e 8 anos $\left(30.000 / \mathrm{mm}^{3}\right)$. As infecções por Anaplasma sp. causaram uma diminuição severa na média da contagem de glóbulos vermelhos, evidenciada em machos (3 milhões $/ \mathrm{mm}^{3}$ ) e fêmeas com menos de 1 ano de idade $\left(3,79\right.$ milhões $\left./ \mathrm{mm}^{3}\right)$.

Tabela 1. Gêneros de hemoparasitas (mórulas típicas) encontrados em cães atendidos no Hospital Veterinário da UFJ entre 2005 e 2017.

\begin{tabular}{|l|l|}
\hline Microorganismos & Porcentagem $(\%)$ \\
\hline Ehrlichia sp. & $9,23(463 / 5015)$ \\
\hline Anaplasma sp. & $4,37(219 / 5015)$ \\
\hline Ehrlichia sp. + Anaplasma sp. & $0,18(9 / 5015)$ \\
\hline TOTAL & $13,78(691 / 5015)$ \\
\hline
\end{tabular}

Tabela 2. Cães infectados com hemoparasitos de acordo com o sexo.

\begin{tabular}{|c|c|c|c|c|}
\hline Sexo & Ehrlichia sp. & Anaplasma sp. & $\begin{array}{c}\text { Ehrlichia sp.+ } \\
\text { Anaplasma sp. }\end{array}$ & Porcentagem (\%) \\
\hline Fêmeas & 252 & 142 & 6 & $57,89(400 / 691)$ \\
\hline Machos & 211 & 77 & 3 & $42,11(291 / 691)$ \\
\hline TOTAL & 463 & 219 & 9 & 691 \\
\hline
\end{tabular}

Stella AE, Silva VLD, Moreira CN, Rezende Junior AS, Lima DA, Schimmunech MS. Aspectos epidemiológicos e hematológicos de cães infectados com Ehrlichia sp e Anaplasma SP em Jataí-GO, Brasil. Vet. e Zootec. 2021; v28: 001-011. 
Tabela 3. Cães infectados de acordo com a faixa etária.

\begin{tabular}{|c|c|c|c|c|c|c|c|}
\hline Idade & $\begin{array}{c}\text { Fêmeas } \\
\text { Ehrlichia sp. }\end{array}$ & $\begin{array}{c}\text { Machos } \\
\text { Ehrlichia sp. }\end{array}$ & $\begin{array}{c}\text { Fêmeas } \\
\text { Anaplasma sp. }\end{array}$ & $\begin{array}{c}\text { Machos } \\
\text { Anaplasma sp. }\end{array}$ & $\begin{array}{c}\text { Fêmeas } \\
\text { Ambos }\end{array}$ & $\begin{array}{c}\text { Machos } \\
\text { Ambos }\end{array}$ & $\begin{array}{c}\text { Porcentagem } \\
(\%)\end{array}$ \\
\hline$\leq 1$ & 82 & 82 & 48 & 27 & 1 & 2 & $35,02(242 / 691)$ \\
\hline $1-8$ & 143 & 114 & 75 & 38 & 3 & 1 & $54,13(374 / 691)$ \\
\hline$\geq 8$ & 27 & 15 & 19 & 12 & 2 & 0 & $10,85(75 / 691)$ \\
\hline Total & 252 & 211 & 142 & 77 & 6 & 3 & 691 \\
\hline
\end{tabular}

Tabela 4. Medianas de parâmetros hematológicos de cães infectados com Ehrlichia sp. e Anaplasma sp.

\begin{tabular}{|l|c|c|c|}
\hline $\begin{array}{c}\text { Parâmetros } \\
\text { hematológicos }\end{array}$ & Ehrlichia sp. & Anaplasma sp. & $\begin{array}{c}\text { Ehrlichia sp. }+ \\
\text { Anaplasma } \text { sp. }\end{array}$ \\
\hline Hematócrito $(\%)$ & 33 & 39 & 37 \\
\hline Hemácias $\left(10^{6} / \mathrm{mm}^{3}\right)$ & 4,82 & 4,34 & 5,5210 \\
\hline Plaquetas $\left(10^{3} / \mathrm{mm}^{3}\right)$ & 114.196 & 222.758 & 116.500 \\
\hline Leucograma $\left(\mathrm{x} 10^{3} / \mu \mathrm{L}\right)$ & 11.046 & 13.454 & 12.150 \\
\hline
\end{tabular}

Tabela 5. Medianas de parâmetros hematológicos de cães infectados com Ehrlichia sp. e Anaplasma sp. de acordo com o sexo e a faixa etária.

\begin{tabular}{|c|c|c|c|c|}
\hline Agente, sexo e faixa etária & $\begin{array}{c}\text { Hematócrito } \\
(\%)\end{array}$ & $\begin{array}{l}\text { Hemácias } \\
\left(10^{6} / \mathrm{mm}^{3}\right) \\
\end{array}$ & $\begin{array}{l}\text { Plaquetas } \\
\left(10^{3} / \mathrm{mm}^{3}\right) \\
\end{array}$ & $\begin{array}{c}\text { Leucograma } \\
\left(\times 10^{3} / \mu \mathrm{L}\right)\end{array}$ \\
\hline Fêmeas/Ehrlichia sp. $<1$ & 31 & 4,02 & 95.110 & 8.658 \\
\hline Fêmeas/Ehrlichia sp. 1-8 & 34 & 6,1 & 123.833 & 11.252 \\
\hline Fêmeas/Ehrlichia sp. $>8$ & 37 & 5,34 & 109.741 & 14.472 \\
\hline Machos/Ehrlichia sp. $<1$ & 30 & 4,32 & 112.747 & 9.706 \\
\hline Machos/Ehrlichia sp. 1-8 & 36 & 4,11 & 116.286 & 10.966 \\
\hline Machos/Ehrlichia sp. $>8$ & 28 & 4,9 & 88.267 & 20.687 \\
\hline Fêmeas/Anaplasma sp. $<1$ & 38 & 3,79 & 247.390 & 11.592 \\
\hline Fêmeas/Anaplasma sp. 1-8 & 39 & 6,1 & 201.060 & 11.470 \\
\hline Fêmeas/Anaplasma sp.> 8 & 34 & 5,14 & 228.057 & 15.032 \\
\hline Machos/Anaplasma sp. $<1$ & 33 & 3 & 213.640 & 12.930 \\
\hline Macho/Anaplasma sp. 1-8 & 38 & 5,68 & 213.553 & 15.599 \\
\hline Macho/Anaplasma sp. $>8$ & 36 & 5,12 & 201.917 & 14.221 \\
\hline $\begin{array}{c}\text { Fêmeas/Ehrlichia } \mathrm{sp} .+ \\
\text { Anaplasma } \mathrm{sp}<1\end{array}$ & 29 & 3 & 40.000 & 16.000 \\
\hline $\begin{array}{c}\text { Fêmeas/Ehrlichia sp. + } \\
\text { Anaplasma sp. 1-8 }\end{array}$ & 37 & 5,2 & 210.000 & 13.150 \\
\hline $\begin{array}{c}\text { Fêmeas/Ehrlichia } \text { sp. }+ \\
\text { Anaplasma } \text { sp. }>8\end{array}$ & 46 & 6,69 & 111.500 & 5.050 \\
\hline $\begin{array}{c}\text { Machos/Ehrlichia sp. }+ \\
\text { Anaplasma } \text { sp. }<1\end{array}$ & 33 & 6,1 & 68.500 & 9.425 \\
\hline $\begin{array}{c}\text { Machos/Ehrlichia } \mathrm{sp} .+ \\
\text { Anaplasma } \text { sp. 1-8 }\end{array}$ & 33 & 4,9 & 30.000 & 15.700 \\
\hline
\end{tabular}

Stella AE, Silva VLD, Moreira CN, Rezende Junior AS, Lima DA, Schimmunech MS. Aspectos epidemiológicos e hematológicos de cães infectados com Ehrlichia sp e Anaplasma SP em Jataí-GO, Brasil. Vet. e Zootec. 2021; v28: 001-011. 


\section{DISCUSSÃO}

A erliquiose monocítica canina, causada pela Erhlichia sp., parece ser altamente endêmica em muitas regiões do Brasil, embora dados de prevalência não estejam disponíveis em muitas delas (15). No período de janeiro de 2005 a dezembro de 2017, 5.015 fichas de exames de análises clínicas veterinárias foram analisadas. Desse total, foram encontrados 9,23 $\%$ de cães portadores de mórula de Ehrlichia sp. e 4,37\% de portadores de Anaplasma sp. Em um estudo no México, Almazan et al. (9) encontraram 31\% de cães positivos para Anaplasma sp. e $10 \%$ para Ehrlichia sp., entre cães saudáveis parasitados com carrapatos, pesquisando 16S rRNA. O resultado para Ehrichia sp. é similar a este estudo, entretanto para Anaplasma $s p$. um número bem maior de animais, no México, se mostraram infectados, isto se deve provavelmente, devido a maior sensibilidade metodológica adotada por aqueles pesquisadores e/ou a diferenças epidemiológicas regionais. Já Bulla et al. (16) em um trabalho realizado no Brasil, encontraram 30,9\% de animais positivos para a presença de um segmento do gene $16 \mathrm{~S}$ rRNA de Ehrlichia canis. Como já dito anteriormente, frequências maiores relatadas muitas vezes estão relacionadas a diferenças metodológicas, o que também parece ocorrer neste caso. Outras pesquisas feitas no Brasil, também apontam variações no número de cães positivos para Ehrichia sp.: 14,6 \% no Maranhão (17), 45\% no Mato Grosso (18), 5,7\% em Minas Gerais (19), 23,7\% também em Minas Gerais (20), 16,4\% no Paraná (21), 24,8\% no Rio de Janeiro (22) e $43 \%$ em São Paulo (23). A frequência de cães positivos para Ehrichia sp. também varia de acordo com os países: 6,4\% na Argélia (3), 7\% (24) e 36,2\% (25) na Turquia, 38,46\% no México (26), $15.3 \%$ na Colômbia (27) e 10,4\% no Paraguai (28). Em relação a positividade para Anaplasma sp., esta frequência também é variável entre os países: 25,2\% na Tunísia (29), 5,45\% na Argélia (3), 34,6\% em Cabo Verde (30), 20,2\% na Colômbia (27) e 10,7\% no Paraguai (28).

Já em uma pesquisa feita na Colômbia, Badillo-Viloria et al. (31) encontraram $6 \%$ de cães positivos para Anaplasma sp., $28 \%$ para Ehrlichia sp. e 2\% para coinfecção (nesta pesquisa $0,18 \%$ ), vale observar que no artigo Colombiano, a técnica utilizada para a investigação de Ehrlichia sp. foi imunológica e com animais desenvolvendo sintomatologia clínica. A coinfecção por hemoparasitas também é uma condição relativamente comum em cães $(5,20,21,23,32,33)$, além disso Baneth et al. (34) sugerem que a infecção com um patógeno pode permitir ou aumentar a invasão ou a sobrevivência celular prolongada de outros. Bem como, Pespane et al. (27) relatam que a coinfecção pode tornar difícil associar um sinal clínico específico a uma determinada doença, transmitida pelo carrapato no cão. Uma vez que Yabsley et al. (35) também citam casos de coinfecção, com até três hemoparasitos diferentes.

Dentre os animais positivos neste estudo, 463 (67\%) apresentaram exclusivamente Ehrlichia sp. Após um período de incubação de 8 a 20 dias, o curso da infecção por Ehrlichia sp. pode ser dividido sequencialmente em fases aguda (2-4 semanas), subclínica (vários meses a anos) e crônica (36), mas a distinção entre essas fases não é simples na doença que ocorre naturalmente (2). Já que a visualização microscópica das mórulas no interior de leucócitos ocorre durante a fase aguda da doença e em poucos casos (37), e como esta identificação na fase crônica é rara, sugere-se uma alta porcentagem de casos de erliquiose/anaplasmose nos animais desta região. Harrus et al. (36) ainda citam que a identificação das mórulas é útil no estabelecimento de um diagnóstico definitivo de erliquiose canina aguda.

Dos 691 cães positivos, 291 eram machos $(42,11 \%)$ e 400 eram fêmeas $(57,89 \%)$, isto é, $7,97 \%$ do total de fêmeas e $5,8 \%$ do total de machos atendidos eram portadores de mórula de Ehrlichia sp. e/ou Anaplasma sp. A ocorrência da erliquiose e/ou anaplasmose canina em fêmeas foi significativamente mais alta. Este resultado confirma os já obtidos na literatura nacional, mostrando uma frequência maior, em fêmeas, destas hemoparasitoses $(19,38)$. No

Stella AE, Silva VLD, Moreira CN, Rezende Junior AS, Lima DA, Schimmunech MS. Aspectos epidemiológicos e hematológicos de cães infectados com Ehrlichia sp e Anaplasma SP em Jataí-GO, Brasil. Vet. e Zootec. 2021; v28: 001-011. 
estudo de Badillo-Viloria et al. (31), Ehrlichia sp. foi mais frequente em machos (63\%) enquanto Anaplasma sp. foi mais encontrado em fêmeas (64\%), nesta pesquisa tanto em machos como em fêmeas Ehrlichia sp. foi mais frequentemente identificada. Como dito anteriormente, estas diferenças também podem ser explicadas pelo uso de técnicas diferentes, mas também devido a condições climáticas e geográficas diversas.

Nos dias de hoje, humanos e animais, principalmente cães, convivem em estreita proximidade. $\mathrm{O}$ que nos leva a pontuar a importância desses agentes na saúde pública, tendo em vista, que já foi relatada a identificação de DNA de E. canis, em amostras de sangue humano (39), bem como de um caso clínico na Turquia (11). Evidências sorológicas sugerem a ocorrência de erliquiose humana no Brasil, entretanto, o agente etiológico ainda não foi identificado (15). Sainz et al. (6) citam que E. canis ainda não é considerado um agente com potencial zoonótico importante.

Muitos animais jovens se mostraram infectados, 35,02\% tinham menos de 1 ano de vida, valores semelhantes aos descritos por Moreira et al. (38). Já Lauzi et al. (30) citam que a frequência de infecção por $E$. canis foi maior em cães com mais de 4 anos, e a infecção por $A$. platys foi maior em cães menores de 2 anos, contrastando com Ojeda-Chi et al. (26) que citam que a frequência de animais com menos de dois anos foi maior para E. canis. Por fim, Sainz et al. (6) citam que a infecção pode ocorrer em qualquer idade, entretanto a positividade é mais comum em cães idosos devido ao maior tempo de exposição. As fêmeas com menos de 1 ano infectadas com Ehrlichia sp. e Anaplasma sp. apresentaram as alterações mais significativas nos aspectos hematológicos, evidenciando anemia e trombocitopenia (Tabela 5). Bulla et al. (16) citam que as contagens de plaquetas podem ser um bom teste de triagem para erliquiose monocítica canina, e que a magnitude da trombocitopenia pode aumentar a confiabilidade do diagnóstico. Erliquiose com trombocitopenia é relatada por Ueno et al. (40); e trombocitopenia e anemia também foram os achados hematológicos mais comumente detectados por Tuna et al. (25). Animais infectados com Anaplasma sp. e Ehrlichia sp., ao mesmo tempo, apresentaram trombocitopenia mais intensa, evidenciada em machos entre $1 \mathrm{e}$ 8 anos (Tabela 5). Já Cetinkaya et al. (4) em um estudo na Turquia, observaram trombocitopenia apenas em animais coinfectados. Gaunt et al. (13) demonstram que a infecção simultânea por $A$. platys e E. canis pode alterar vários parâmetros fisiopatológicos em cães infectados experimentalmente.

As infecções por Anaplasma sp. causaram uma diminuição severa na média da contagem de glóbulos vermelhos, evidenciada em machos e fêmeas com menos de 1 ano de idade (Tabela 5). Enquanto Silva et al. (21) relatam, que a infecção por E. canis teve relação com anemia e com trombocitopenia, enquanto a infecção por A. platys apresentou relação apenas com trombocitopenia. Entretanto, Bouzouraa et al. (23) em seu estudo com Anaplasma sp. citam alterações como trombocitopenia, anemia, leucocitose e leucopenia. Gianopoulus et al. (41) citam que a infecção está associada a várias alterações discretas quantitativas e qualitativas do leucograma, indicativas de inflamação concomitante, estimulação antigênica e estresse. Borin et al. (19) em seu estudo observaram anemia, predominando o tipo normocítica normocrômica, desvio nuclear de neutrófilos para a esquerda e eosinopenia. Como demonstrado neste trabalho e nos estudos acima citados, uma baixa contagem de plaquetas, é a principal alteração hematológica nestas doenças (42). Além disso, existem mudanças significativas no proteoma sérico de cães com erliquiose com modificações nas proteínas relacionadas com a resposta de fase aguda (43).

\section{CONCLUSÕES}

Os resultados encontrados neste estudo reforçam a alta prevalência e endemicidade desses agentes em cães nesta região do estado de Goiás, Brasil. Isto mostra um grande desafio

Stella AE, Silva VLD, Moreira CN, Rezende Junior AS, Lima DA, Schimmunech MS. Aspectos epidemiológicos e hematológicos de cães infectados com Ehrlichia sp e Anaplasma SP em Jataí-GO, Brasil. Vet. e Zootec. 2021; v28: 001-011. 
para a saúde animal e humana, pois existe a probabilidade de transmissão para os humanos. As fêmeas, com menos de um ano e coinfectadas, apresentaram as alterações mais significativas nos aspectos hematológicos. Concluindo, nossos resultados suportam a hipótese de infecções naturais por Erlichia sp. e Anaplasma sp. em cães atendidos no Hospital Veterinário da UFJ na cidade de Jataí-GO, sugerindo que na população canina estudada, houve prevalência de infecção subclínica (assintomática). Estes resultados epidemiológicos podem ser considerados para o controle e prevenção das doenças transmitidas por carrapatos, em uma região pouco estudada, e ao que parece, com cães desempenhando um papel de reservatório.

\section{REFERÊNCIAS}

1. Gaff HD, Kocan KM, Sonenshine DE. Tick-borne rickettsioses II (Anaplasmataceae) In: Sonenshine DE, Roe RM, editors. Biology of ticks. 2nd ed. Oxford: Oxford University; 2014. v. 2, p. 251-77.

2. Mylonakis ME, Konstantina NT. Canine monocytic ehrlichiosis: an update on diagnosis and treatment. Acta Vet. 2017;67(3):299-317.

3. Dahmani M, Loudahi A, Mediannikov O, Fenollar F, Raoult, D, Davoust, B. Molecular detection of Anaplasma platys and Ehrlichia canis in dogs from Kabylie, Algeria. Ticks Tick Borne Dis. 2015;6(2):198-203.

4. Cetinkaya H, Matur E, Akyazi I, Ekiz EE, Aydin L, Toparlak M. Serological and molecular investigation of Ehrlichia spp. and Anaplasma spp. in ticks and blood of dogs, in the Thrace Region of Turkey. Ticks Tick Borne Dis. 2016;7(5):706-14.

5. Cardoso L, Tuna J, Vieira L, Yisaschar-Mekuzas Y, Baneth G. Molecular detection of Anaplasma platys and Ehrlichia canis in dogs from the North of Portugal. Vet $\mathrm{J}$. 2010;183(2):232-3.

6. Sainz Á, Roura X, Miró G, Estrada-Peña A, Kohn B, Harrus S, et al. Guideline for veterinary practitioners on canine ehrlichiosis and anaplasmosis in Europe. Parasit Vectors. 2015;8(1):75.

7. Aguiar DM, Rodrigues FP, Ribeiro MG, Dos Santos B, Muraro LS, Taques IIGG, et al. Uncommon Ehrlichia canis infection associated with morulae in neutrophils from naturally infected dogs in Brazil. Transbound Emerg Dis. 2020;67 Suppl 2:135-41.

8. Geromichalou A, Faixová Z. Haematopathological changes in dogs affected with Ehrlichia canis in Lesvos. Folia Vet. 2017;61(2):44-9.

9. Almazán C, Gonzalez-Alvarez VH, de Mera IGF, Cabezas-Cruz A, Rodríguez-Martínez R, de la Fuente J. Molecular identification and characterization of Anaplasma platys and Ehrlichia canis in dogs in Mexico. Ticks Tick Borne Dis. 2016;7(2):276-83.

10. Bowman D, Little SE, Lorentzen L, Shields J, Sullivan MP, Carlin EP. Prevalence and geographic distribution of Dirofilaria immitis, Borrelia burgdorferi, Ehrlichia canis, and Anaplasma phagocytophilum in dogs in the United States: results of a national clinicbased serologic survey. Vet Parasitol. 2009;160(1-2):138-48. 
11. Emiroğlu M, Çelebi B. First report of human ehrlichiosis in Turkey. Turk J Pediatr. 2019;61(2):267-70.

12. Neer TM, Breitschwerdt EB, Greene RT, Lappin MR. Consensus statement on ehrlichial disease of small animals from the infectious disease study group of the ACVIM. J Vet Intern Med. 2002;16(3):309-15.

13. Gaunt SD, Beall MJ, Stillman BA, Lorentzen L, Diniz PPVP, Chandrashekar R, et al. Experimental infection and co-infection of dogs with Anaplasma platys and Ehrlichia canis: hematologic, serologic and molecular findings. Parasit Vectors. 2010;3(1):33.

14. Nakaghi ACH, Machado RZ, Costa MT, André MR, Baldani CD. Canine ehrlichiosis: clinical, hematological, serological and molecular aspects. Cienc Rural. 2008;38(3):76670 .

15. Vieira RFDC, Biondo AW, Guimarães AMS, Santos APD, Santos RPD, Dutra LH, et al. Ehrlichiosis in Brazil. Rev Bras Parasitol Vet. 2011;20(1):1-12.

16. Bulla C, Takahira RK, Araújo JP, Trinca LA, Lopes RS, Wiedmeyer CE. The relationship between the degree of thrombocytopenia and infection with Ehrlichia canis in an endemic area. Vet Res. 2004;35(1):141-6.

17. Costa APD, Costa FB, Labruna MB, Silveira I, Moraes-Filho J, Soares, et al. A serological and molecular survey of Babesia vogeli, Ehrlichia canis and Rickettsia spp. among dogs in the state of Maranhão, northeastern Brazil. Rev Bras Parasitol Vet. 2015;24(1):28-35.

18. Costa JS, Melo ALT, Witter R, Pacheco TA, Chitarra CS, Carvalho ITS, et al. Molecular detection of Ehrlichia canis in Rhipicephalus sanguineus (sl) ticks in dogs and their domestic environment in Cuiaba, MT, Brazil. Braz J Vet Res Anim Sci. 2019;56(2):e153661.

19. Borin S, Crivelenti LZ, Ferreira FA. Aspectos epidemiológicos, clínicos e hematológicos de 251 cães portadores de mórula de Ehrlichia spp. naturalmente infectados. Arq Bras Med Vet Zootec. 2009;61(3):566-71.

20. Fonseca JP, Bruhn FRP, Ribeiro MJM, Hirsch C, Rocha CMBM, Guedes E, et al. Hematological parameters and seroprevalence of Ehrlichia canis and Babesia vogeli in dogs. Cienc Anim Bras. 2017;118:1-9.

21. Silva GCFD, Benitez ADN, Girotto A, Taroda A, Vidotto MC, Garcia JL. Occurrence of Ehrlichia canis and Anaplasma platys in household dogs from northern Parana. Rev Bras Parasitol Vet. 2012;21(4):379-85.

22. Paulino PG, Pires MS, Silva CB, Peckle M, Costa RL, Vitari GV, et al. Epidemiology of Ehrlichia canis in healthy dogs from the Southeastern region of the state of Rio de Janeiro, Brazil. Prev Vet Med. 2018;159:135-42.

Stella AE, Silva VLD, Moreira CN, Rezende Junior AS, Lima DA, Schimmunech MS. Aspectos epidemiológicos e hematológicos de cães infectados com Ehrlichia sp e Anaplasma SP em Jataí-GO, Brasil. Vet. e Zootec. 2021; v28: 001-011. 
23. Oliveira TMF, Furuta PI, Carvalho DD, Machado RZ. Study of cross-reactivity in serum samples from dogs positive for Leishmania sp., Babesia canis and Ehrlichia canis in enzyme-linked immunosorbent assay and indirect fluorescent antibody test. Rev Bras Parasitol Vet. 2008;17(1):7-11.

24. Elitok B, Ungur B. Prevalence of Ehrlichia canis infection in Uşak and investigation of clinical, hematological and biochemical signs in infected dogs. Int Biol Biomed J. 2016;2(4):134-9.

25. Tuna GE, Bakirci S, Dinler C, Karagenç T, Ulutaş B. Monocytic Ehrlichiosis in aegean region dogs: clinical and haematological findings. Atatürk Üniversitesi Vet Bil Derg. 2019; 14(1): 8-14.

26. Ojeda-Chi MM, Rodriguez-Vivas RI, Esteve-Gasent MD, de León AAP, Modarelli JJ, Villegas-Perez SL. Ehrlichia canis in dogs of Mexico: prevalence, incidence, coinfection and factors associated. Comp Immunol Microbiol Infect Dis. 2019;67:101351.

27. Pesapane R, Foley J, Thomas R, Castro LR. Molecular detection and characterization of Anaplasma platys and Ehrlichia canis in dogs from northern Colombia. Vet Microbiol. 2019;233:184-9.

28. Pérez-Macchi S, Pedrozo R, Bittencourt P, Müller A. Prevalence, molecular characterization and risk factor analysis of Ehrlichia canis and Anaplasma platys in domestic dogs from Paraguay. Comp Immunol Microbiol Infect Dis. 2019;62:31-9.

29. M'ghirbi Y, Ghorbel A, Amouri M, Nebaoui A, Haddad S, Bouattour A. Clinical, serological, and molecular evidence of ehrlichiosis and anaplasmosis in dogs in Tunisia. Parasitol Res. 2009;104(4):767-74.

30. Lauzi S, Maia JP, Epis S, Marcos R, Pereira C, Luzzago C, et al. Molecular detection of Anaplasma platys, Ehrlichia canis, Hepatozoon canis and Rickettsia monacensis in dogs from Maio Island of Cape Verde archipelago. Ticks Tick Borne Dis. 2016;7(5):964-9.

31. Badillo-Viloria M, Díaz-Pérez A, Orozco-Sánchez C, Lavalle-Galvis R. Infection by Ehrlichia canis and Anaplasma sp. in dogs attended in veterinary clinics, Barranquilla, Colombia. Rev MVZ Cordoba. 2017;22 Supl:6023-33.

32. Rojas A, Rojas D, Montenegro V, Gutiérrez R, Yasur-Landau D, Baneth G. Vector-borne pathogens in dogs from Costa Rica: first molecular description of Babesia vogeli and Hepatozoon canis infections with a high prevalence of monocytic ehrlichiosis and the manifestations of co-infection. Vet Parasitol. 2014;199(3-4):121-8.

33. Bouzouraa T, René-Martellet M, Chêne J, Attipa C, Lebert I, Chalvet-Monfray K, et al. Clinical and laboratory features of canine Anaplasma platys infection in 32 naturally infected dogs in the Mediterranean basin. Ticks Tick Borne Dis. 2016;7(6):1256-64.

34. Baneth G, Harrus S, Gal A, Aroch I. Canine vector-borne co-infections: Ehrlichia canis and Hepatozoon canis in the same host monocytes. Vet Parasitol. 2015;208(1-2):30-4.

Stella AE, Silva VLD, Moreira CN, Rezende Junior AS, Lima DA, Schimmunech MS. Aspectos epidemiológicos e hematológicos de cães infectados com Ehrlichia sp e Anaplasma SP em Jataí-GO, Brasil. Vet. e Zootec. 2021; v28: 001-011. 
35. Yabsley MJ, McKibben J, Macpherson CN, Cattan PF, Cherry NA, Hegarty BC, et al. Prevalence of Ehrlichia canis, Anaplasma platys, Babesia canis vogeli, Hepatozoon canis, Bartonella vinsonii berkhoffii, and Rickettsia spp. in dogs from Grenada. Vet Parasitol. 2008;151(2-4):279-85.

36. Harrus S, Waner T, Neer TM. Ehrlichia canis infection. In: Sykes J, Greene C. Infectious diseases of the dog and cat. 4th ed. St. Louis: Elsevier Saunders; 2012.

37. Mylonakis ME, Koutinas AF, Breitschwerdt EB, Hegarty BC, Billinis CD, Leontides LS, et al. Chronic canine ehrlichiosis (Ehrlichia canis): a retrospective study of 19 natural cases. J Am Anim Hosp Assoc. 2004;40(3):174-84.

38. Moreira SM. Estudo retrospectivo (1998-2001) da erliquiose canina em Belo Horizonte: avaliação clínica e laboratorial de infecções experimentais [dissertação]. Belo Horizonte: Escola de Veterinária, Universidade Federal de Minas Gerais; 2001.

39. Bouza-Mora L, Dolz G, Solórzano-Morales A, Romero-Zuñiga JJ, Salazar-Sánchez L, Labruna MB, et al. Novel genotype of Ehrlichia canis detected in samples of human blood bank donors in Costa Rica. Ticks Tick Borne Dis. 2017; 8(1):36-40.

40. Ueno TE, Aguiar DM, Pacheco RC, Richtzenhain LJ, Ribeiro MG, Paes AC, et al. Ehrlichia canis em cães atendidos em hospital veterinário de Botucatu, Estado de São Paulo, Brasil. Rev Bras Parasitol Vet. 2009;18(3):57-61.

41. Gianopoulos A, Mylonakis ME, Theodorou K, Christopher MM. Quantitative and qualitative leukocyte abnormalities in dogs with experimental and naturally occurring acute canine monocytic ehrlichiosis. Vet Clin Pathol. 2016;45(2):281-90.

42. Harrus S, Waner T. Diagnosis of canine monocytotropic ehrlichiosis (Ehrlichia canis): an overview. Vet J. 2011;187(3):292-6.

43. Escribano D, Cihan H, Martínez-Subiela S, Levent P, Kocaturk M, Aytug N, et al. Changes in serum proteins in dogs with Ehrlichia canis infection. Microb Pathog. 2017;113:34-9.

Recebido em: 04/11/2020

Aceito em: 12/03/2021

Stella AE, Silva VLD, Moreira CN, Rezende Junior AS, Lima DA, Schimmunech MS. Aspectos epidemiológicos e hematológicos de cães infectados com Ehrlichia sp e Anaplasma SP em Jataí-GO, Brasil. Vet. e Zootec. 2021; v28: 001-011. 